The Egyptian Journal of Hospital Medicine (October 2017) Vol. 69 (4), Page 2278-2285

\title{
Management of Diabetic Ketoacidosis in Patients with Diabetes Type I Basam Zuhaer Sindi ${ }^{1}$, Naif Mishkhas Alazwari ${ }^{2}$, Abdulaziz Mohammed Khateeb ${ }^{2}$, Aqil Abdulmonem Alzaher ${ }^{3}$, Mohammad Abdulaziz M Alkhawajah ${ }^{3}$, Atheer Khalid Almutairi ${ }^{3}$, Najd Abdulrahman Al- Mutairi $^{3}$, Zainab Reda Alghanim ${ }^{3}$, Ameera Ahmad Nemer ${ }^{3}$, Hazim Abdulkarim Khatib ${ }^{2}$ \\ 1 Umm Alqura University, 2 King Abdulaziz University, 3 Imam Abdulrahman Bin Faisal University Corresponding Author: Basam zuhaer sindi, email:Basam.sindi@gmail.com
}

\begin{abstract}
Background: DKA is the leading cause of mortality among pediatric age and young adults with T1D, responsible for almost $50 \%$ of all fatalities in diabetic patients younger than 24 years of age. Goals of DKA management include optimization of volume status, hyperglycemia and ketoacidosis, electrolyte abnormalities, and potential precipitating factors. Methodology: we conducted this review using a comprehensive search of MEDLINE, PubMed, and EMBASE, January 1987, through February 2017. The following search terms were used: ketoacidosis, management of diabetic ketoacidosis, type I diabetic patients' emergency complication, fluid replacement in DKA, insulin therapy approach. Aim: in this review, we aim at evaluating the various ways of approaching patients who suffer from type-one diabetes during ketoacidosis and keto-acidotic coma. We will try to understand the triggers and pathophysiology behind this condition, and explore ways to prevent them. Conclusion: prompt diagnoses, aggressive treatment, and education of patient and their care providers about prevention strategies must be implemented. Also, more studies are required in the area of preventing health complications due to these types of diseases to effectively manage DKA in the future.
\end{abstract}

Keywords: diabetic ketoacidosis approach, insulin therapy in diabetic ketoacidosis, fluid replacement in diabetic ketoacidosis.

\section{INTRODUCTION}

Diabetic ketoacidosis (DKA) is a common cause of morbidity and mortality in type-1 diabetics; though, infrequently it also occurs in type-2 diabetics. Type 1 diabetes mellitus (T1D) affects children and adults worldwide, with an increasing incidence between young people. The incidence of DKA has risen by $30 \%$ through the past decade, with greater than 140,000 hospital admissions every year in the United States alone [1]. Patients with the highest risk comprised those with high hemoglobin A1C, longer diabetes length, adolescents, and females. DKA is the chief cause of mortality amid children and young adults with T1D, adding up to nearly $50 \%$ of all deaths in a young diabetic patient of age less than 24 years ${ }^{[2]}$. Inside United States, the overall inpatient DKA mortality is only $1 \%$, but an advanced rate is stated for elderly patients who also have other life-threatening illnesses ${ }^{[1]}$.

There were 140,000 hospitalizations for DKA with an average length of stay of about 3.4 days in the year 2009. The annual cost of DKA hospitalizations adds up to 2.4 billion US dollars ${ }^{[3]}$. The therapeutic aims of DKA management comprise optimization of:
1. Volume status.

2. Hyperglycemia and ketoacidosis.

3. Electrolyte abnormalities.

4. Potential precipitating factors ${ }^{[4]}$.

The majority of patients with DKA present to the emergency room. DKA-related ICU and hospital deaths have stayed steady for nearly fifteen years and no improvement in mortality was observed ${ }^{[5]}$.

In this review, we aimed evaluation of the various aspects to approach a patient with type-one diabetes during ketoacidosis and keto-acidotic coma.

We tried to understand the triggers and pathophysiology behind such conditions, and learn how to prevent them.

\section{METHODOLOGY}

- Data Sources and Search terms

We conducted this review using a comprehensive search of MEDLINE, PubMed, and EMBASE, January 1987, through February 2017. The following search terms were used: ketoacidosis, management of diabetic ketoacidosis, type I diabetic patients' emergency complication, fluid replacement in DKA, insulin therapy approach 


\section{- Data Extraction}

Two reviewers have independently reviewed the studies, abstracted data, and disagreements were resolved by consensus. Studies were evaluated for quality and a review protocol was followed throughout.

The study was done after approval of ethical board of King Abdulaziz University.

\section{The study was done after approval of ethical board of King Abdulaziz university.}

\section{MANAGEMENT OF DKA IN TYPE 1 Diabetes Mellitus}

DKA is described by the presence of hyperglycemia, ketosis, and metabolic acidosis. This happens as a consequence of a relative or absolute insulin deficiency and high amount of counterregulatory hormones such as glucagon, catecholamines, and cortisol causing hyperglycemia, glycosuria, hyperosmolarity, and dehydration of variable intensity. Fluid deficits can be up to $10 \%$ of total body weight ${ }^{[6]}$. Glycosuria starts an osmotic diuresis, which causes significant deficits in fluid and electrolytes especially sodium, potassium, calcium, magnesium, and phosphate. Dehydration and hyperglycemia leads to hypertonicity resulting in an efflux of water from the inside the cell to the extracellular space ${ }^{[7]}$. It is accompanied by a potassium efflux from the inside of the cell. Additionally, DKA is a hypercoagulable state so it possibly leads to fatal complications such as stroke, disseminated intravascular coagulation, myocardial infarction. There is also an accumulation of $\beta$ hydroxybutyrate and acetoacetate, which results in a high anion gap metabolic acidosis. The typical symptoms of uncontrolled diabetes that causes development of DKA are polyuria, polydipsia, polyphagia, weight loss, fatigue, vomiting, and abdominal pain ${ }^{[8]}$.

\section{Precipitating Causes}

DKA is reported to be the first presentation of diabetes in about $15 \%$ to $20 \%$ of adults and in

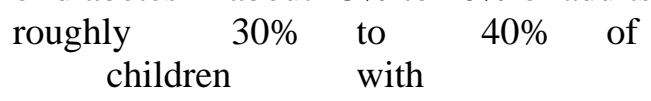

T1D. Infection is the most probable cause of DKA worldwide. Poor adherence to insulin treatment, however, is the biggest precipitating factor of DKA in young patients with T1D in the United States ${ }^{[8]}$. A recent report from a hospital in Atlanta says that insulin discontinuation was responsible for $56 \%$ of patients' presentation of DKA, while in $78 \%$ of patients it was accountable for multiple DKA episodes. Other triggers of DKA included infectious causes which are 14, and noninfectious illnesses which make 4\%. Non-infectious causes include acute myocardial infarction, alcohol use, neurovascular accidents, and pancreatitis ${ }^{[9]}$. Other non-infectious causes are psychological which include depression and eating disorders. These have been noted in up to $20 \%$ of recurrent episodes of DKA, especially in young patients. Insulin pump malfunction can cause. However, in the newer improved technology of pump, this is not a common event. Medications can also be a cause of DKA, for example, those that alter carbohydrate metabolism, including glucocorticoids, beta-blockers, thiazide diuretics, atypical antipsychotics ${ }^{[10]}$, and certain chemotherapeutic agents. Adding to the list of drugs is the sodium glucose co-transporter 2 inhibitors, which are a new class of oral anti-diabetic agents, have been associated with DKA in patients with both type I and type II diabetes ${ }^{[11]}$.

\section{Pathophysiology}

The pathophysiologic mechanisms for DKA development include two main mechanisms: substantial insulin deficiency, and increased amount of counter-regulatory. This leads to increased hepatic glucose production by increased hepatic gluconeogenesis and glycogenolysis. There is additionally reduced glucose utilization by the peripheral tissues, especially in muscle. Low insulin causes activation of hormone-sensitive lipase and increases breakdown of triglycerides into free fatty acids. In the liver, the free fatty acids are oxidized to ketone bodies which are stimulated by glucagon ${ }^{[12]}$. The imbalance of insulin and glucagon leads to an increased glucagon/insulin ratio which lowers the activity of malonyl coenzyme A. Malonyl coenzyme A modulates movement of free fatty acid inside the hepatic mitochondria and fatty acid oxidation occurs. There is increased production of ketone bodies. They are mainly acetoacetate and $\beta$ hydroxybutyrate which are two strong acids. This leads to reduction of bicarbonate and causes metabolic acidosis ${ }^{[13]}$.

Many experiments and clinical studies showed that the development of hyperglycemia and ketoacidosis leads to an inflammatory state. It is marked by an increase of pro-inflammatory cytokines and oxidative stress markers. Severe hyperglycemiainduced macrophages produce proinflammatory 
cytokines like tumor necrosis factoralpha, IL-6, IL-1 $\beta$, and C-reactive protein. They further cause more insulin secretion impairment and additionally reduce the insulin sensitivity ${ }^{[14]}$. Free fatty acid also increases resistance to insulin and causes endothelial dysfunction because of decreased nitric oxide production. The entire inflammatory response, oxidative stress and production of reactive oxygen species cause capillary perturbation and damage membranes, proteins, and DNA $^{[6]}$.

\section{Diagnosis}

Signs and Symptoms: Patients presenting with DKA have a brief clinical course marked by fatigue and polyuria, polydipsia, and weight loss, which are the classical symptoms of hyperglycemia.

Gastrointestinal complaints are often present such as diffuse abdominal pain (46\%), and nausea and vomiting $(66 \%)^{[15]}$. Lethargy and stupor $(31 \%)$ can be present, however less than $25 \%$ lose consciousness. On physical examination, the most common signs are the signs of dehydration, such as dry mucous membranes with poor skin turgor, and sometimes, tachycardia, or hypotension. Patients in DKA can have Kussmaul respirations and a fruity breath odor ${ }^{[16]}$.

\section{Laboratory Investigations}

The American Diabetes Association classifies DKA by severity as mild, moderate, or severe which is based on the grade of acidosis as well as decrease in bicarbonate amount, and changed sensorium. Most DKA patients present with mild to moderate severity (blood glucose greater than $250 \mathrm{mg} / \mathrm{dL}$, bicarbonate $10-18 \mathrm{mEq} / \mathrm{L}$, arterial $\mathrm{pH}>7.3$, ketones bodies in the blood or urine, and increased anion gap $>12^{[17]}$.

While the bulk of patients present with plasma glucose levels of $>250 \mathrm{mg} / \mathrm{dL}$, few patients show only mild elevations in plasma glucose levels and so that is called 'euglycemic DKA'. This phenomenon has been noted in pregnant patients, in prolonged starvation, patients with high alcohol intake, and those who have recently been started on SGLT-2 inhibitor use ${ }^{[18]}$.

The key in diagnosis is an elevation in circulating total blood ketone along with high anion gap metabolic acidosis $>12$. Ketonemia assessment is done by the nitroprusside reaction in urine or serum. It shows a semiquantitative estimate of ketone levels ${ }^{[19]}$. The nitroprusside test has a high sensitivity, but it cannot detect severity of ketoacidosis because it fails to recognize the presence of $\beta$-hydroxybutyrate, which is the most important metabolic product of ketoacidosis. Keeping that in mind, it is preferred to do a direct measurement of serum $\beta$ hydroxybutyrate for diagnosis ${ }^{[20]}$.

\section{Management of DKA}

DKA management includes [4]: -fluid and electrolyte correction, -insulin therapy, -treatment of any underlying causes, and -monitoring of possible complications

Treatment objectives include correcting dehydration, reducing hyperglycemia, normalizing hyperosmolality, correcting electrolyte imbalance, and identifying and treating the precipitating event. The typical time to resolution is $10-18$ hours for DKA. During the treatment, lab measurements of glucose, electrolytes, venous $\mathrm{pH}$, anion gap, and bicarbonate must be repeated every 2 to 4 hours ${ }^{[19]}$.

\section{Hydration}

Intravenous (IV) fluids are the most important aspect while treating hyperglycemic emergencies. Treatment with IV fluids alone can expand the intravascular volume, restoring renal perfusion, and can decrease insulin resistance by lowering circulating counter-regulatory hormone levels. Isotonic saline $(0.9 \% \mathrm{NaCl})$ is the ideal solution which is set at rate of $500-1000 \mathrm{~mL} / \mathrm{h}$ in the first $2-4$ hours ${ }^{[7]}$.

The logic behind intensive hydration in DKA is to avoid hypoperfusion, correct the hyperglycemia, and improve insulin therapy responses. A study comparing 2 IV fluid regimens with sodium chloride and ringer lactate showed no significant difference in time to resolve DKA; however the ringer lactate took considerably longer time to correct hyperglycemia. Following IV volume repletion, the rate of infusion of isotonic saline must be decreased to $250 \mathrm{~mL} / \mathrm{h}$, or isotonic saline can be replaced with $0.45 \%$ saline based on the serum sodium concentration and state of hydration. After the plasma glucose level has reached around $200 \mathrm{mg} / \mathrm{dL}$, the IV fluids should hold 5\% to $10 \%$ of dextrose to permit insulin administration until ketonemia is improved. This also avoids hypoglycemia. Rapid hydration, however, has a potential risk of cerebral edema, which is seen rarely in pediatric population ${ }^{[21]}$. 


\section{Potassium Correction}

Initially, patients with DKA can have mild to moderate hyperkaliemia, although there is a total body deficit of potassium. The deficiency of insulin and metabolic acidosis lead to efflux of potassium. Therefore, serum potassium levels may appear to be normal or increased, but the patients have a low overall potassium level in the body. Insulin therapy promotes the movement of potassium back into the intracellular compartment thus lowering the serum potassium level [22]. Accordingly, potassium replacement should be started once the concentration in blood is $<5.2 \mathrm{mEq} / \mathrm{L}$ in order to maintain a vital level of $4-5 \mathrm{mEq} / \mathrm{L}$. It is advisable advices to administer $20-30 \mathrm{mEq}$ of potassium per liter of fluids. This amount is sufficient for most patients, except in case of patient with chronic renal failure where lower doses are required for patients ${ }^{[23]}$. For patients with serum potassium levels of $<3.3 \mathrm{mEq} / \mathrm{L}$, insulin administration may cause severe hypokalemia resulting in muscle weakness and increased risk of cardiac arrhythmias. Potassium replacement should be given at a rate of $10-20$ $\mathrm{mEq} / \mathrm{h}$ and insulin therapy must be delayed in such patients until the potassium level reaches to $>3.3$ $\mathrm{mEq} / \mathrm{L}^{[24]}$.

\section{Bicarbonate Therapy}

Role of bicarbonate is controversial. Few say that correcting ketosis with insulin is satisfactory for acidosis. Others suggest that bicarbonate therapy is warranted given the complications of severe metabolic acidosis. Routine administration of bicarbonate did not prove to improve clinical outcomes, in terms of time to resolution, hospital stay, or even mortality. Normally it is only recommended in patients with very severe acidosis with a $\mathrm{pH}<6.9^{[4]}$. Bicarbonate can potentially increase the risk of deadly complications as hypokalemia and cerebral edema. Therefore in lifethreatening cases, clinical guidelines endorse the administration of $50-100 \mathrm{mmol}$ of sodium bicarbonate in an isotonic solution until $\mathrm{pH}$ becomes $>$ 6.9. In milder cases, bicarbonate therapy is not indicated ${ }^{[25]}$.

\section{Insulin Therapy}

Administration of insulin is the backbone of DKA management as it lowers the serum glucose by various mechanisms, such as, inhibiting glucose production endogenously, and by increasing peripheral use. Insulin further inhibits lipolysis, production of ketone bodies, and glucagon secretion, in this manner lessening the production of ketoacidosis ${ }^{[26]}$.

Regular insulin in continuous IV infusion is the treatment of choice. Most of the protocols advice administering of $0.1 \mathrm{U} / \mathrm{kg}$ of body weight bolus first, and then followed by continuous IV insulin infusion at $0.1 \mathrm{U} / \mathrm{kg} /$ hour until blood glucose reaches $200 \mathrm{mg} / \mathrm{dL}$. After that the dose is decreased by onehalf $(0.05$ $\mathrm{U} / \mathrm{kg} /$ hour) while rate is set between 0.020 .05 $\mathrm{U} / \mathrm{kg} / \mathrm{hour}$ and $5 \%$ dextrose is added, to maintain glucose concentrations between $140200 \mathrm{mg} / \mathrm{dL}$. The therapy is stopped with resolution of ketoacidosis ${ }^{[20}$; 27].

Studies have shown that the giving doses of rapid insulin analogs (Lispro and Aspart) subcutaneous every couple of hours can act as an effective alternative to the regular insulin in IV infusion with respect to time to DKA resolution. In this method, patients are given an initial bolus of $0.2-0.3 \mathrm{U} / \mathrm{kg}$ and then $0.1-0.2 \mathrm{U} / \mathrm{kg}$ every 1 to 2 hours, until glucose gets below $250 \mathrm{mg} / \mathrm{dL}$. After that, insulin is reduced by a half to $0.05 \mathrm{U} / \mathrm{kg}$ hourly, or

$0.01 \mathrm{U} / \mathrm{kg}$ every 2 hours until the DKA resolution. This procedure of administrating insulin promises a safe yet effective treatment in the emergency department without needing ICU care

[17].

Rapid- acting insulin given as intramuscular injections is also effective in the treating DKA. This route, however, it is more painful than the other routes of administration, and might increase bleeding risk of patients on anticoagulation therapy. At the same time, rapid-acting subcutaneous insulin analogues are not advised for DKA patients with arterial hypotension, severe DKA, or with hyperosmolar hyperglycemic state $^{[20]}$.

\section{Phosphate and Magnesium}

Like potassium, phosphate levels may seem to be normal or elevated at presentation, but with treatment, it declines because phosphate goes in the intracellular space. Studies do not show a benefit from phosphate replacement therapy ${ }^{[28]}$. On the contrary, it can be linked to hypocalcemia and hypomagnesemia. However, phosphate deficiency is associated with fatigue of muscle, rhabdomyolysis, respiratory failure, 
hemolysis, and arrhythmia. Therefore, if phosphate level is $<1.0 \mathrm{mg} / \mathrm{dL}$, or when these complications occur, its replacement is recommended. The therapy is given as $20-30 \mathrm{mEq}$ of potassium phosphate to the IV fluid ${ }^{[29]}$.

DKA further causes magnesium drop, which can ends in paresthesia, spasm, seizures, tremor, and cardiac arrhythmia. It is replaced if below $1.2 \mathrm{mg} / \mathrm{dL}$, or if symptomatic ${ }^{[28]}$.

\section{Resolution}

DKA resolution is defined when glucose levels are below $250 \mathrm{mg} / \mathrm{dL}$, the anion gap normalizes, venous $\mathrm{pH}$ rises above 7.3 , and serum bicarbonate is equal to or above $18 \mathrm{mEq} / \mathrm{L}$. Abrupt cessation of the insulin may result is rebound hyperglycemia, ketogenesis, and metabolic acidosis because of short half-life. Subcutaneous basal insulin is given 2 hours before terminating the IV insulin administration. If basal insulin analogues are being used, then they must be administered 3 to 4 hours before discontinuing insulin drip as their onset of action is more delayed than NPH insulin ${ }^{[30]}$.

For patients who have never taken insulin before, total daily insulin $0.5-0.6 \mathrm{U} / \mathrm{kg}$ can be given. Patients who have poor oral intake must get basal insulin alone or, otherwise, may be kept on an insulin drip until they can eat. Patients with known T1D should be restarted on their former regimens. The regimen might need adjustment if there is a history of frequent hypoglycemia, hyperglycemia, or increased $\mathrm{HbA} 1 \mathrm{c}$. Multi-dose insulin schedules with basal insulin along with rapid-acting insulin analogues are the favored insulin plan T1D patients with DKA ${ }^{[31]}$.

\section{Complications of DKA}

The most common complication during treatment is hypoglycemia as reported in 5\% - 25\% of DKA patients. Risk factors of hypoglycemia are lack of regular monitoring, failing to reduce rate of insulin infusion, and the use of dextrose solutions when blood glucose drops below $200 \mathrm{mg} / \mathrm{dL}$. Unfortunately, many patients suffering from hypoglycemia do not reveal manifestations of sweating, nervousness, fatigue, tachycardia, hunger ${ }^{[32]}$. This is why monitoring blood glucose frequently, every 1 to 2 hours, is required. Acute negative results of hypoglycemia comprise of seizures, arrhythmias, and altered consciousness.
The second most common complication is hypokalemia during DKA management. As explained above, during admission serum potassium level is usually elevated. However, when insulin is administered, the serum concentration of potassium will invariably go down due to increased intracellular potassium uptake by the peripheral tissues ${ }^{[33]}$. In order to prevent hypokalemia, potassium must be replaced with IV when its concentration falls below 5.2 $\mathrm{mEq} / \mathrm{L}$. If the patients' serum potassium level is less than $3.3 \mathrm{mEq} / \mathrm{L}$, insulin must be held and IV potassium replacement should be given immediately, until serum potassium rises.

Cerebral edema is reported in nearly $1 \%$ of pediatric DKA with a high mortality rate of $20 \%$ to $40 \%$, while it is rare in adults. Its pathogenesis is not completely understood. Disruption of the bloodbrainbarrier was found in autopsy of fatal cases of edema ${ }^{[34]}$. There is proportionality between the degree of dehydration and the degree of edema, but it has no relation with osmolality, osmotic changes at the time of treatment, or rate of administration of fluid or insulin. Cerebral edema frequently occurs 4 to 12 hours after treatment was initiated, but may also happen up to 24 to 48 hours after the initiation of treatment. Suggested management includes mannitol 0.5 to $1 \mathrm{~g} / \mathrm{kg}$ IV over 20 minute administration, and it is repeat if there is no response for 30 minutes. Alternative to mannitol, hypertonic saline (3\%), to $10 \mathrm{~mL} / \mathrm{can}$ be given for 30 minutes, especially if patient is refractory to mannitol therapy. A cranial CT scan must be obtained in order to rule out other possible causes of neurologic deficits, such as thrombosis and infarction, hemorrhage ${ }^{[35]}$.

Acute renal failure and shock can also be a possible complication of DKA. Other uncommon complications include rhabdomyolysis, pneumomediastinum, prolonged corrected QT interval, pulmonary edema, thrombosis, stroke, and memory loss with low cognitive function in children ${ }^{[32]}$.

\section{Prevention of DKA}

Medication noncompliance is a foremost reason of DKA among both newly diagnosed and recurrent episodes of DKA. Sadly, in 50\% of such DKA attacks, patients mentioned an inability to have the funds for buying medication or to pay for transportation resulting in discontinuation of medication. This requires assistance programs to deliver insulin to patients and decrease lapses in treatment in a cost-effective method to lessen the 
amount of hospitalization for such emergencies [36]. Multidisciplinary approaches with help of medical diabetes educators who are easily accessible to the patients has shown to diminish the figure of hospitalizations. Systems-based methods to decrease preventable etiologies of DKA may signify a central next step in lowering costs and refining patient care

[37].

Physicians should inform and teach patients and their caregivers about recognition of early symptoms. Patients and caregivers must be acquainted with altering insulin through times of sickness. Handy home measurement of urine/ blood ketones with glucometer might permit for earlier detection of DKA and improve outcomes. Patients who are on insulin pump must learn their pump settings, and always have a prescription for basal insulin if the pump ever fails to work ${ }^{[38]}$.

\section{Euglycemic Diabetic Ketoacidosis}

There is a sub-classification in which the serum glucose levels are within the normal limits. This condition is known as euglycemic DKA (EDKA). EDKA is defined as a condition with high anion gap metabolic acidosis, presence of serum and urine ketones, and a serum glucose level of $<250 \mathrm{mg} / \mathrm{dL}{ }^{[15]}$. The primary mechanism of EDKA is reduced hepatic production of glucose during fasting state, or increased urinary excretion of glucose because of counter-regulatory hormones. When a diabetic patient is open to any eliciting factor for DKA, but is also fasting or starving, or is on the insulin treatment, the liver will enter a state of glycogen depletion, and only producing a lesser amount of glucose. While on the other side, lipolysis and fatty acid production takes place, leading to disproportionate ketone body production. Common causes of EDKA reported in studies are low intake of calorie, fasting, vomiting, diarrhea, pregnancy, cocaine intoxication, insulin pump use, and use of SGLT2 inhibitors ${ }^{[39]}$.

Dehydration typically stimulates the development of hyperglycemia. Dehydration performs as a stimulus for added glucagon secretion causing lipolysis and ketone production, along with decreased glucose production, thus resulting in EDKA. In the course of insulin deficiency, dehydration likewise increases the secretion of counter-regulatory hormones, which additionally exacerbates EDKA ${ }^{[40]}$.
Diagnosis of EDKA is problematic as it is a diagnosis of exclusion. Other forms of ketoacidosis and increased anion gap metabolic acidosis must to be ruled out. After being diagnosed, management of EDKA is nearly parallel to the management of DKA. The backbone of management includes rapid correction of dehydration using IV fluids ${ }^{[41]}$. The second step in the managing is insulin drip along with a dextrose solution until the anion gap, and bicarbonate levels stabilize. Routine testing of urine for ketones as well as arterial blood gas analysis to evaluate anion gap are necessary till the values stabilize. If diagnosed promptly and managed aggressively with fluids and insulin, EDKA can be reversed easily, thus decreasing morbidity and mortality ${ }^{[42]}$.

\section{CONCLUSION}

As we have noted above in the studies that DKA is the leading cause of mortality among pediatric age and young adults with T1D, responsible for almost $50 \%$ of all fatalities in diabetic patients younger than 24 years of age, prompt diagnoses, aggressive treatment, and teaching of patient and their care providers about prevention strategies must be implemented. Also, more studies in the area of preventing complications of treatment must be done to effectively manage DKA in future.

\section{REFERENCES}

1. Maynard GA, Holdych J, Kendall H, Harrison K, Montgomery PA and Kulasa K (2017): Improving Glycemic Control Safely in Critical Care Patients: A Collaborative Systems Approach in Nine Hospitals. Endocr Pract., 23: 583-593.

2. Seyoum B and Berhanu P (2007): Profile of diabetic ketoacidosis in a predominantly African American urban patient population. Ethn Dis., 17: 234-237.

3. Kitabchi $A E$ and Nyenwe EA (2006): Hyperglycemic crises in diabetes mellitus: diabetic ketoacidosis and hyperglycemic hyperosmolar state. Endocrinol Metab Clin North Am., 35: 725-751.

4. Glaser $\mathbf{N}$ (2006): New perspectives on the pathogenesis of cerebral edema complicating diabetic ketoacidosis in children. Pediatr Endocrinol Rev., 3: 379-386.

5. Venkatesh B, Pilcher D, Prins J, Bellomo R, Morgan TJ and Bailey $M$ (2015): Incidence and outcome of adults with diabetic ketoacidosis admitted to ICUs in Australia and New Zealand. Crit Care, 19: 451.

6. Misra S and Oliver NS (2015): Diabetic ketoacidosis in adults. BMJ., 351: h5660. 
7. Eledrisi MS, Alshanti MS, Shah MF, Brolosy B and Jaha N (2006): Overview of the diagnosis and management of diabetic ketoacidosis. Am J Med Sci., 331: 243-251.

8. Kitabchi AE, Umpierrez GE, Miles JM and Fisher JN (2009): Hyperglycemic crises in adult patients with diabetes. Diabetes Care, 32: 1335-1343.

9. Kumar P, Sakwariya A, Sultania AR and Dabas R (2017): Hypertriglyceridemia-induced acute pancreatitis with diabetic ketoacidosis: A rare presentation of type 1 diabetes mellitus. J Lab Physicians, 9: 329-331.

10. Vuk A, Baretic M, Osvatic MM, Filipcic I, Jovanovic N and Kuzman MR (2017): Treatment of Diabetic Ketoacidosis Associated With Antipsychotic Medication: Literature Review. J Clin

Psychopharmacol., 37: 584-589.

11. Jabbour S, Seufert J, Scheen A, Bailey CJ, Karup $C$ and Langkilde AM (2017): Dapagliflozin in patients with type 2 diabetes mellitus: a pooled analysis of safety data from Phase $2 b / 3$ clinical trials. Diabetes Obes Metab., DOI: 10.1111/dom.13124

12. Kitabchi AE, Umpierrez GE, Murphy MB and Kreisberg RA (2006): Hyperglycemic crises in adult patients with diabetes: a consensus statement from the American Diabetes Association. Diabetes Care, 29: 2739-2748.

13. Andrade-Castellanos CA, Colunga-Lozano LE, Delgado-Figueroa $\mathbf{N}$ and Gonzalez-Padilla DA (2016): Subcutaneous rapid-acting insulin analogues for diabetic ketoacidosis. Cochrane Database Syst., doi: 10.1002/14651858.

14. Umpierrez GE, Smiley D and Kitabchi AE (2006): Narrative review: ketosis-prone type 2 diabetes mellitus. Ann Intern Med., 144: 350-357.

15. Rawla P, Vellipuram AR, Bandaru SS, Pradeep Raj J. Euglycemic diabetic ketoacidosis: a diagnostic and therapeutic dilemma. Endocrinol Diabetes Metab Case Rep 2017; 2017.

16. Klocker AA, Phelan H, Twigg SM and Craig ME (2013): Blood beta-hydroxybutyrate vs. urine acetoacetate testing for the prevention and management of ketoacidosis in Type 1 diabetes: a systematic review. Diabet Med., 30: 818-824.

17. Parkin CG, Graham C and Smolskis J (2017):

Continuous Glucose Monitoring Use in Type 1 Diabetes: Longitudinal Analysis Demonstrates Meaningful Improvements in HbA1c and Reductions in Health Care Utilization. J Diabetes Sci Technol., 11: 522-528.

18. Misra S and Oliver NS (2015): Utility of ketone measurement in the prevention, diagnosis and management of diabetic ketoacidosis. Diabet Med., 32: $14-23$.

19. Dreschfeld J (1886): The Bradshawe Lecture on Diabetic Coma. Br Med J., 2: 358-363.
20. Okiro JO, Mc Hugh C and Abdalla A (2017): Is it safe to acutely discontinue insulin therapy in patients with chronic hyperglycaemia starting GLP-1R agonists? BMJ Case Rep., doi:10.1136/bcr-2017220437

21. Winter WE, Maclaren NK, Riley WJ, Clarke DW, Kappy MS and Spillar RP (1987): Maturity-onset diabetes of youth in black Americans. N Engl J Med., 316: 285-291.

22. Beigelman PM (1973): Potassium in severe diabetic ketoacidosis. Am J Med., 54: 419-420.

23. Ferreira JP, Hamui M, Torrents M, Carrano R, Ferraro M and Toledo I (2017): The Influence of Chloride for the Interpretation of Plasma Bicarbonate During the Treatment of Diabetic Ketoacidosis. Pediatr Emerg Care, DOI: 10.1097/PEC.0000000000001245

24. Yamada H, Funazaki S, Kakei M, Hara $K$ and Ishikawa SE (2017): Diabetic ketoacidosis producing extreme hyperkalemia in a patient with type 1 diabetes on hemodialysis. Endocrinol Diabetes Metab Case Rep., DOI: 10.1530/EDM-17-0068

25. Taylor SI, Blau JE and Rother KI (2015): SGLT2 Inhibitors May Predispose to Ketoacidosis. J Clin Endocrinol Metab., 100: 2849-2852.

26. Martensson J, et al. (2017): Intensity of early correction of hyperglycaemia and outcome of critically ill patients with diabetic ketoacidosis. Crit Care Resusc., 19: 266-273.

27. Fazeli Farsani S, Brodovicz K, Soleymanlou N, Marquard J, Wissinger E and Maiese BA (2017): Incidence and prevalence of diabetic ketoacidosis (DKA) among adults with type 1 diabetes mellitus (T1D): a systematic literature review. BMJ Open, 7: e016587.

28. Cadacio C, Pham PT, Bhasin R, Kamarzarian A and Pham PC (2017): Multiple Electrolyte and Metabolic Emergencies in a Single Patient. Case Rep Nephrol., doi: 10.1155/2017/4521319.

29. Shen $\mathbf{T}$ and Braude $\mathbf{S}$ (2012): Changes in serum phosphate during treatment of diabetic ketoacidosis: predictive significance of severity of acidosis on presentation. Intern Med J., 42: 1347-1350.

30. Datye KA, et al. (2017): Timing of Meal Insulin and Its Relation to Adherence to Therapy in Type 1 Diabetes.

$$
\text { J Diabetes Sci Technol., doi: }
$$

10.1177/1932296817728525

31. Barski L, Brandstaetter E, Sagy I and Jotkowitz A (2017): Basal insulin for the management of diabetic ketoacidosis. Eur J Intern Med., doi:

10.1016/j.ejim.2017.08.025

32. Bialo SR, Agrawal S, Boney CM and Quintos JB (2015): Rare complications of pediatric diabetic ketoacidosis. World J Diabetes, 6: 167-174. 
33. Satyarengga $M$, Zubatov $\mathbf{Y}$, Frances $\mathrm{S}$, Narayanswami $G$ and Galindo RJ (2017): Glycogenic Hepatopathy: A Complication of Uncontrolled Diabetes. AACE Clin Case Rep., 3: e255-e259.

34. Brown TB (2004): Cerebral oedema in childhood diabetic ketoacidosis: is treatment a factor? Emerg Med J., 21: 141-144.

35. Silverstein J, et al. (2005): Care of children and adolescents with type 1 diabetes: a statement of the American Diabetes Association. Diabetes Care, 28: 186212.

36. Basarir H, et al. (2016): Cost-Effectiveness of Structured Education in Children with Type-1 Diabetes Mellitus. Int J Technol Assess Health Care, 32: 203-211.

37. Vyas C, Dalal L, Talaviya $P$ and Saboo B (2017): Multiple educational programs improves glycemic control, quality of life with diminishing the impact of diabetes in poorly controlled type 1 diabetics. Diabetes Metab Syndr., doi: 10.1016/j.dsx.2017.04.011.
38. Guo J (2017): Smartphone-Powered Electrochemical Dongle for Point-of-Care Monitoring of Blood betaKetone. Anal Chem., 89: 8609-8613.

39. Abu-Abed Abdin A, Hamza M, Khan MS and Ahmed A (2016): Euglycemic Diabetic Ketoacidosis in a Patient with Cocaine Intoxication. Case Rep Crit Care, doi: 10.1155/2016/4275651

40. Andrews TJ, Cox RD, Parker C and Kolb J (2017): Euglycemic Diabetic Ketoacidosis with Elevated Acetone in a Patient Taking a Sodium-Glucose Cotransporter-2 (SGLT2) Inhibitor. J Emerg Med., 52: 223-226.

41. Bader $\mathbf{N}$ and Mirza L (2016): Euglycemic Diabetic Ketoacidosis in a 27 year-old female patient with type1Diabetes treated with sodium-glucose cotransporter-2 (SGLT2) inhibitor Canagliflozin. Pak J Med Sci., 32: 786-788.

42. Kelmenson DA, Burr K, Azhar Y, Reynolds P, Baker CA and Rasouli N (2017): Euglycemic Diabetic

Ketoacidosis With Prolonged Glucosuria Associated

With the Sodium-Glucose Cotransporter-2 Canagliflozin. J Investig Med High Impact Case Rep.,

doi: $10.1177 / 2324709617712736$. 